LBL-36744 - UCB-95/03

NUB-3117

LPTHE-Orsay 95/14

hep-th/9504143

\title{
Dynamical Supersymmetry Breaking and the Linear Multiplet ${ }^{\star}$
}

\author{
P. Binétruy, ${ }^{a}$ M.K. Gaillard ${ }^{b}$ and T.R. Taylor ${ }^{c}$ \\ ${ }^{a}$ Laboratoire de Physique Théorique et Hautes Energies, ${ }^{\dagger}$ Université Paris-Sud, \\ F-91405 Orsay, France \\ ${ }^{b}$ Department of Physics and Theory Group, Lawrence Berkeley Laboratory, \\ University of California, Berkeley 94720, U.S.A. \\ ${ }^{c}$ Department of Physics, Northeastern University, Boston, MA 02115, U.S.A.
}

\begin{abstract}
We analyze gaugino condensation in the presence of a dilaton and an antisymmetric tensor field, with couplings reminiscent of string theories. The degrees of freedom relevant to a supersymmetric description of the effective theory below the scale of condensation are discussed in this context.
\end{abstract}

${ }^{\star}$ Research supported in part by the Director, Office of High Energy and Nuclear Physics, Division of High Energy Physics of the U.S. Department of Energy under contract DE-AC03-76SF00098 and in part by the National Science Foundation under grants PHY-90-21139 and PHY-93-06906.

${ }^{\dagger}$ Laboratoire associé au CNRS-URA-D0063.

April 1995 


\section{Introduction}

The dilaton and antisymmetric tensor fields that are found among massless string modes are believed to play an important role in the dynamics of supersymmetry breaking. Together with their supersymmetric partners, they form a linear supermultiplet that is described by a vector superfield $L$ satisfying the constraint $\bar{D}^{2} L=D^{2} L=0$, in rigid supersymmetry notation. Thus, the vector component $\mathcal{B}^{m}$ satisfies

$$
\partial_{m} \mathcal{B}^{m}=0
$$

and is therefore constrained to describe the field strength of a Kalb-Ramond field (two-index antisymmetric tensor field): $\mathcal{B}^{m}=\epsilon^{m n p q} \partial_{n} b_{p q}$.

The generation of masses for the dilaton and the two-form presents one of the most important problems faced by superstring phenomenology. It must have a non-perturbative nature since the linear multiplet remains massless to all orders in the string loop expansion. This problem has been addressed before in the context of dynamical supersymmetry breaking induced by gaugino condensation. The standard strategy is to first perform a duality transformation replacing the linear superfield by a chiral one, and thus replacing the antisymmetric tensor by a pseudoscalar axion. A superpotential for the chiral superfield can then be derived in the framework of effective field theory. However the analysis of the induced potential is plagued by serious difficulties such as the absence of a stable vacuum and a vanishing axion mass.

Although the equivalence between linear and chiral multiplets is certainly valid for onshell amplitudes, it is not clear that it holds in off-shell quantities like non-perturbative effective potentials. It is therefore very important to consider gaugino condensation with the dilaton and antisymmetric tensor, without recurring to any duality transformation. In particular, it has been known for quite a long time that a non-zero mass for the antisymmetric tensor can be generated by couplings to a vector or to a three-form (three-index 
antisymmetric tensor field). In this paper, we show that in the effective theory of gaugino condensation, these extra degrees of freedom are supplied by gauge-singlet composite fields. The effective theory is written in terms of a single vector multiplet which contains these composite fields in addition to the degrees of freedom contained in the linear multiplet.

The antisymmetric tensor field plays a very important role in the anomaly cancellation of heterotic superstring theory. As a consequence, in the Lagrangian describing the massless string modes, the linear multiplet always appears in the gauge-invariant combination $L-\Omega$, where $\Omega$ is the Chern-Simons (real) superfield. The Yang-Mills part of $\Omega$ is defined by $\bar{D}^{2} \Omega=-k W^{\alpha} W_{\alpha}$ with $k$ a normalisation factor, so that

$$
\bar{D}^{2}(L-\Omega)=k W^{\alpha} W_{\alpha}
$$

where $W^{\alpha}$ is the usual gauge field-strength chiral superfield.

In the effective theory of gaugino condensation, we replace the superfield $k W^{\alpha} W_{\alpha}$ by an effective chiral superfield $U$ describing the condensate degrees of freedom [1]. As a consequence of the Bianchi identities in the Yang-Mills sector, this superfield must derive from a real "prepotential" $V$, that is

$$
\bar{D}^{2} V=U
$$

By comparison with eq.([1.2), we see that the single vector superfield $V$ describes the "elementary" degrees of freedom of the linear multiplet together with the "composite" degrees of freedom of the gauge singlet bound states. This vector supermultiplet will be the central focus of the following analysis.

The essence of the non-perturbative analysis is contained in a superpotential term for $U=\bar{D}^{2} V$ that is dictated by the anomaly structure of the underlying theory [1]. The full effective action is then completed by replacing the gauge invariant combination $L-\Omega$ by $V$ in the original action.

The next section is an illustration of these ideas in the context of global supersymmetry. 
The problem of the determination of the form of the kinetic terms for the effective degrees

of freedom cannot be solved at this level using only symmetry arguments. We therefore consider in section 3 the context of superstring theories where higher derivative terms in the action of the underlying theory may provide the right kinetic terms for the dynamical degrees of freedom.

\section{The global supersymmetry case.}

In this section, we start with global supersymmetry and a toy model that reproduces some of the basic properties of the more realistic supergravity models to be considered in what follows. We show in particular that gaugino condensation in presence of a dilaton field $\mathcal{C}$ and an antisymmetric tensor $b_{m n}$ induces a non-trivial potential for the dilaton field, in agreement with the results obtained previously in the dual formulation.

Let us consider a general vector supermultiplet $V$. We will write its component field expansion in superspace as

$$
\begin{aligned}
V & =\mathcal{C}+i \theta \chi-i \bar{\theta} \bar{\chi}+\theta^{2} h+\bar{\theta}^{2} h^{*}+\theta \sigma^{m} \bar{\theta} \mathcal{B}_{m} \\
& +i \theta^{2} \bar{\theta}\left(\bar{\eta}+\frac{i}{2} \bar{\sigma}^{m} \partial_{m} \chi\right)-i \bar{\theta}^{2} \theta\left(\eta+\frac{i}{2} \sigma^{m} \partial_{m} \bar{\chi}\right)+\frac{1}{2} \theta^{2} \bar{\theta}^{2}\left(D+\frac{1}{2} \square \mathcal{C}\right)
\end{aligned}
$$

The standard way to proceed from there is to impose a constraint in superspace, the socalled linear multiplet constraint $D^{2} V=\bar{D}^{2} V=0$ which yields in component form

$$
\partial^{m} \mathcal{B}_{m}=0
$$

together with the vanishing of all auxiliary fields. Eq.(2.2) is the Bianchi identity which ensures that $\mathcal{B}_{m}$ is dual to the field strength of an antisymmetric tensor:

$$
\mathcal{B}_{m}=\epsilon_{m n p q} \partial^{n} b^{p q}
$$

We will instead obtain the Bianchi identity (2.2) as an equation of motion. 
Let us indeed consider the following Lagrangian for $V$

$$
\mathcal{L}=\int d^{4} \theta K(V)+\left(\int d^{2} \theta W\left(\bar{D}^{2} V\right)+\text { h.c. }\right),
$$

where $K$ is a real function of $V$ whereas $W$ is an analytic function of the chiral superfield $\bar{D}^{2} V$, which will turn out to be our prepotential field.

Since we are mainly interested in applying our ideas to the string dilaton-axion-dilatino system, we will make the following choices

$$
K(V)=\log V, \quad W(U)=-\frac{b}{4} U \log U,
$$

where $U=\bar{D}^{2} V$. The form of the superpotential is dictated by the anomaly structure of the underlying theory and $b$ is proportional to the one-loop beta function coefficient.

The Lagrangian (2.4) can in fact be obtained through a duality transformation as follows. Consider the alternative Lagrangian:

$$
\mathcal{L}=\int d^{4} \theta(K(V)+(V-\Xi)(S+\bar{S}))+\left(\int d^{2} \theta W(\Delta)+\text { h.c. }\right)
$$

where $S$ and $\Delta$ are chiral superfields which are further constrained by requiring that $\Delta$ derives from the real prepotential $\Xi$; in other words,

$$
\begin{aligned}
& \Delta=\bar{D}^{2} \Xi, \\
& \bar{\Delta}=D^{2} \Xi .
\end{aligned}
$$

We will see later that such a chiral superfield naturally arises when one tries to describe a 3-form supermultiplet in supersymmetry or supergravity. For the time being, in the context of gaugino condensation, $\Delta$ can be thought as a relic $\operatorname{Tr} W^{\alpha} W_{\alpha}$ and $\Xi$ as a relic Chern-Simons superfield. The two are obviously connected. This identification becomes more transparent if one minimizes with respect to $V$, in which case one finds simply

$$
V=-\frac{1}{S+\bar{S}}
$$


and the Lagrangian (2.6) can be written as

$$
\mathcal{L}=\int d^{4} \theta[-\log (S+\bar{S})]+\left(\int d^{2} \theta(4 S \Delta+W(\Delta))+\text { h.c. }\right) .
$$

Note that we have not included a kinetic term for $\Delta$. In other words, we do not consider the dynamics of composite fields in this first approach. We will return below to this question.

One can alternatively minimize the Lagrangian (2.6) with respect to $S$, which yields

$$
\begin{aligned}
& \bar{D}^{2} V=\bar{D}^{2} \Xi=\Delta, \\
& D^{2} V=D^{2} \Xi=\bar{\Delta},
\end{aligned}
$$

in which case one recovers the original Lagrangian (2.4).

Let us now write the Lagrangian (2.4) in terms of the component fields (we will disregard here the fermion fields):

$$
\begin{aligned}
\mathcal{L}_{\text {bos }}= & -\frac{1}{4 \mathcal{C}^{2}} \partial^{m} \mathcal{C} \partial_{m} \mathcal{C}+\frac{1}{4 \mathcal{C}^{2}} \mathcal{B}^{m} \mathcal{B}_{m}+\left(\frac{1}{2 \mathcal{C}}+b(1+\log |4 h|)\right)(D+\square \mathcal{C}) \\
& -\frac{1}{\mathcal{C}^{2}}|h|^{2}+i \frac{b}{2} \log \frac{h^{*}}{h} \partial^{m} \mathcal{B}_{m}
\end{aligned}
$$

Writing $h \equiv \rho e^{i \omega}$, we obtain by minimizing with respect to the phase $\omega$ precisely the Bianchi identity

$$
\partial^{m} \mathcal{B}_{m}=0
$$

which ensures that $\mathcal{B}_{m}$ is dual to the field strength of an antisymmetric tensor according to (2.3). The term $\mathcal{B}^{m} \mathcal{B}_{m}$ in the Lagrangian is therefore a kinetic term for this tensor field.

Minimizing with respect to $D$ yields

$$
\rho=\frac{1}{4 e} e^{-\frac{1}{2 b c}}
$$

The Lagrangian now reads

$$
\begin{aligned}
\mathcal{L} & =-\frac{1}{4 \mathcal{C}^{2}} \partial^{m} \mathcal{C} \partial_{m} \mathcal{C}+\frac{1}{4 \mathcal{C}^{2}} \mathcal{B}^{m} \mathcal{B}_{m}-V(\mathcal{C}) \\
V(\mathcal{C}) & =\frac{1}{16 e^{2} \mathcal{C}^{2}} e^{-\frac{1}{b \mathcal{C}}}
\end{aligned}
$$


Finally, minimizing (2.11) with respect to $\rho$ yields

$$
D=\frac{1}{8 b e^{2} \mathcal{C}^{2}} e^{-\frac{1}{b \mathcal{C}}}
$$

The fact that we are left with an auxiliary field $D$ is another departure from the standard linear multiplet treatment, which is known not to include any auxiliary field.

Let us note that our treatment is very dependent on the form (2.5) of the superpotential $W$. It can easily be shown that only such a form - up to a constant term - allows one to recover the Bianchi identity (2.2) through the minimization condition on the phase of the $h$ field.

In a more realistic model which includes kinetic terms for the composite degrees of freedom, the simple Bianchi identity (2.12) no longer holds. It is replaced by

$$
\partial^{m} \mathcal{B}_{m}=\frac{1}{8}{ }^{*} \Phi
$$

where ${ }^{*} \Phi$ is related to the field strength of a rank-3 antisymmetric tensor field $\Gamma_{n p q}$, remnant of the Chern-Simons form in the effective theory:

$$
{ }^{*} \Phi=\frac{1}{3 !} \epsilon_{m n p q} \partial^{m} \Gamma^{n p q}
$$

Indeed, in this context, the fact that the scalar field $U$ derives from a real prepotential $V$ through (1.3) allows us to interpret its degrees of freedom as those of a 3-form supermultiplet [2, 3, 4]. Such a supermultiplet is precisely constructed with the help of a scalar field $U$ which satisfies the constraint巴

$$
D^{2} U-\bar{D}^{2} \bar{U}=2 i^{*} \Phi
$$

\footnotetext{
${ }^{1}$ Such a supermultiplet is constructed from a super 3 -form gauge potential $\Gamma_{A B C}(A, B, C$ being vector or spinor indices). Its field strength $\Phi_{A B C D}$ is constrained by $\Phi_{\alpha \beta \gamma A}=0(\alpha, \beta, \gamma$ dotted or undotted spinor indices). The analysis of the Bianchi identity $d \Phi=0$ shows that all the other components are expressed in terms of a single chiral superfield $U$ defined by: $\Phi^{\dot{\alpha} \dot{\beta}}{ }_{a b}=16\left(\bar{\sigma}_{a b} \epsilon\right)^{\dot{\alpha} \dot{\beta}} U$. For example, $\Phi_{a b c d}=-\epsilon_{a b c d}{ }^{*} \Phi$ with $2 i^{*} \Phi=D^{2} U-\bar{D}^{2} \bar{U}$.
} 
where ${ }^{*} \Phi$ is the gauge invariant field strength of the 3 -form as in (2.17). One can show that the relation (2.18) holds only for a chiral superfield $U$ deriving from a real prepotential such as in $U=\bar{D}^{2} V$.

The solution to (2.16) is

$$
\mathcal{B}_{m}=\frac{1}{3 !} \epsilon_{m n p q}\left(\frac{1}{8} \Gamma^{n p q}+\partial^{n} b^{p q}+\partial^{q} b^{n p}+\partial^{p} b^{q n}\right)
$$

where $b^{p q}$ is a 2 -form. This 2 -form can be gauged away by performing a gauge transformation on the 3-form:

$$
\Gamma^{n p q} \rightarrow \Gamma^{n p q}+\partial^{[n} \Lambda^{p q]}
$$

With this choice of gauge, the vector $\mathcal{B}_{m}$ can be interpreted as a field dual to a 3 -form,

$$
\mathcal{B}_{m}=\frac{1}{8 \cdot 3 !} \epsilon_{m n p q} \Gamma^{n p q}
$$

In order to be more specific, we need to write the kinetic term for the composite degrees of freedom. The problem is that symmetry considerations do not restrict this term in any significant way and, as long as we do not place ourselves in the context of a given theory (see next section), we have little information about such a term. We will therefore choose the generic form

$$
\mathcal{L}_{k i n}=\alpha \int d^{4} \theta V^{n}\left(\bar{D}^{2} V D^{2} V\right)^{p}
$$

The full Lagrangian now reads in component form:

$$
\begin{aligned}
\mathcal{L}= & -f(\rho, \mathcal{C}) \partial^{m} \mathcal{C} \partial_{m} \mathcal{C}-\alpha \mathcal{C}^{n}\left(16 \rho^{2}\right)^{p}\left(\frac{\partial^{m} \rho \partial_{m} \rho}{\rho^{2}}+\partial^{m} \omega \partial_{m} \omega\right) \\
& +4 \alpha p^{2} \mathcal{C}^{n}\left(16 \rho^{2}\right)^{p-1}\left(\partial^{m} \mathcal{B}_{m}\right)^{2}+f(\rho, \mathcal{C}) \mathcal{B}^{m} \mathcal{B}_{m}-\left[b+\alpha n p \mathcal{C}^{n-1}\left(16 \rho^{2}\right)^{p}\right] \mathcal{B}^{m} \partial_{m} \omega \\
& -4 f(\rho, \mathcal{C}) \rho^{2}+4 \alpha p^{2} \mathcal{C}^{n}\left(16 \rho^{2}\right)^{p}(D+\square \mathcal{C})^{2} \\
& +\left[\frac{1}{2 \mathcal{C}}\left(1+\alpha n(2 p+1) \mathcal{C}^{n}\left(16 \rho^{2}\right)^{p}\right)+b(1+\log (4 \rho))\right](D+\square \mathcal{C})
\end{aligned}
$$

where

$$
f(\rho, \mathcal{C})=\frac{1}{4 \mathcal{C}^{2}}\left[1-\alpha n(n-1) \mathcal{C}^{n}\left(16 \rho^{2}\right)^{p}\right]
$$


In eq. (2.23), the auxiliary field $D$ can be eliminated in a simple way by using its equations of motion.

In eq.(2.23), the vector field degrees of freedom are represented by a 3 -form, as in eq.(2.21). The field equation obtained by varying $\Gamma_{n p q}$ can be expressed in terms of the scalar field:

$$
a \equiv \frac{1}{2} \alpha p^{2} \mathcal{C}^{n}\left(16 \rho^{2}\right)^{p-1 *} \Phi
$$

It reads

$$
\partial^{k}\left(\frac{2 \mathcal{C}^{2}\left[2 \partial_{k} a+\left(b+\alpha n p \mathcal{C}^{n-1}\left(16 \rho^{2}\right)^{p}\right) \partial_{k} \omega\right]}{1-\alpha n(n-1) \mathcal{C}^{n}\left(16 \rho^{2}\right)^{p}}\right)=\frac{a}{4 \alpha p^{2} \mathcal{C}^{n}\left(16 \rho^{2}\right)^{p-1}}
$$

This has the form of an equation of motion for the scalar $a$, mixed with the phase $\omega$ at the level of the kinetic terms, i.e.,

$$
\mathcal{L}_{k i n}=-\frac{\mathcal{C}^{2}}{1-\alpha n(n-1) \mathcal{C}^{n}\left(16 \rho^{2}\right)^{p}}\left(2 \partial^{m} a+\left(b+\alpha n p \mathcal{C}^{n-1}\left(16 \rho^{2}\right)^{p}\right) \partial^{m} \omega\right)^{2}
$$

and whose non-derivative interactions are described by the potential

$$
v(a)=\frac{a^{2}}{4 \alpha p^{2} \mathcal{C}^{n}\left(16 \rho^{2}\right)^{p-1}} .
$$

Note that $a$ represents a gauge-invariant degree of freedom from the point of view of the 3 -form gauge transformation (2.20). We therefore interpret $a$ as an axion-like degree of freedom associated with the linear multiplet of the fundamental theory. The potential term (2.28) thus represents a mass generation for the corresponding antisymmetric tensor field. And the equation of motion for $\Gamma_{n p q}$ is dual to the equation of motion of a massive axion.

The Lagrangian (2.23) therefore describes the interaction of two - dilatonic and axionic fundamental degrees of freedom $\mathcal{C}$ and $a \sim \partial^{m} \mathcal{B}_{m}$ and two - dilatonic and axionic - effective degrees of freedom $h$ and $\omega$, describing gaugino condensates. The fundamental axionic degree of freedom can also be interpreted as an effective gauge condensate $\left\langle F^{m n} \tilde{F}_{m n}\right\rangle$.

It is quite interesting to note that the couplings in (2.23) responsible for the mass generation of the axionic field $a$ were proposed earlier [5, 6] on the basis of a parallel with 
the Schwinger model in two dimensions. This connection sheds some light on the reason why no massless particle appears in the physical spectrum of the theory.

The minimum of the full scalar potential occurs at $a=0$. Further minimization with respect to $\rho$ and $\mathcal{C}$ yields

$$
\begin{aligned}
\left(1+\alpha n(2 p+1) \mathcal{C}^{n}\left(16 \rho^{2}\right)^{p}\right)+2 b \mathcal{C}(1+\log (4 \rho)) & =0 \\
f(\rho, \mathcal{C})=\frac{1}{4 \mathcal{C}^{2}}\left[1-\alpha n(n-1) \mathcal{C}^{n}\left(16 \rho^{2}\right)^{p}\right] & =0
\end{aligned}
$$

The first equation implies zero vacuum expectation value of the auxiliary field $D$, while the second leads to a vanishing normalisation factor of the kinetic energy term for the $\mathcal{C}$ field, as can be seen from (2.23). The minimization procedure thus leads in this simple model to a singular point of the field configuration. We now turn to more realistic configurations where the form of the kinetic terms can be inferred from the structure of an underlying fundamental theory.

\section{Local Supersymmetry}

\subsection{Minimal Terms}

We will now consider a locally supersymmetric model for the prepotential $V$ describing the linear multiplet together with the composite degrees of freedom. The Lagrangian will be constructed by using the formalism of superconformal supergravity, with $N=1$ Poincaré gauge fixing constraints imposed on the chiral compensator [7]. Recall that in this formalism, the multiplets are characterized by their dimensions and $U(1)$ charges: real vector superfields are neutral whereas the charges of chiral superfields are equal to their dimensions. The chiral compensator $\Sigma$ has dimension 1 while the real linear multiplet, hence also the prepotential $V$, has dimension 2. The fact that $V$ carries a non-zero dimension, which is a consequence of its gravitational origin, is very useful for discussing various limiting cases of the effective theory. In general, a locally supersymmetric action can 
be constructed from an F-component of a chiral superfield of dimension 3 or from a Dcomponent of a real vector multiplet of dimension 2 .

Once the gauge-invariant combination $L-\Omega$ is replaced by the vector superfield $V$, the tree-level kinetic terms of the dilaton become

$$
-\left.(\Sigma \bar{\Sigma})^{3 / 2}\left(\frac{V}{2}\right)^{-1 / 2}\right|_{\mathrm{D}}=\left.\sqrt{2} \mathcal{P}\left[(\Sigma \bar{\Sigma})^{3 / 2} V^{-1 / 2}\right]\right|_{\mathrm{F}}+\text { h.c. }
$$

where $\mathcal{P}$ is the chiral projection operator - a generalization of $-\bar{D}^{2} / 2$ to the local case. 7 It has dimension 1 and charge 3 .

As already mentioned, the form of the non-perturbative superpotential is dictated by the anomaly structure of the underlying gauge theory. It gives rise to the interaction term

$$
-\left.b \mathcal{P}[V] \ln \left(-2 \mathcal{P}[V] / \Sigma^{3}\right)\right|_{\mathrm{F}}+\text { h.c. }
$$

In the following discussion, we will be interested mostly in the bosonic terms in the Lagrangian, in particular in the scalar potential. The bosonic components of $V$ are, as in the global case, the dilaton $\mathcal{C}$, the vector $\mathcal{B}^{m}$ and two auxiliary fields, $h$ as an F-component and $D$ as a D-component. We also define

$$
\Pi \equiv \sqrt{2} \mathcal{P}\left[(\Sigma \bar{\Sigma})^{3 / 2} V^{-1 / 2}\right]
$$

a chiral superfield of dimension 3 .

The most convenient gauge choice for the scalar component $\sigma$ of the compensator is

$$
\sigma^{3}=\sqrt{\mathcal{C} / 2}
$$

The bosonic part of the Lagrangian

$$
\mathcal{L}=\left.\Pi\right|_{\mathrm{F}}-\left.b \mathcal{P}[V] \ln \left(-2 \mathcal{P}[V] / \Sigma^{3}\right)\right|_{\mathrm{F}}+\text { h.c. }
$$

\footnotetext{
${ }^{2}$ Hence a factor -2 rescaling on the global superspace measure $d^{2} \theta$ is necessary in order to recover the conventions of ref. [7] used in this section.
} 
is given in this gauge by

$$
\begin{aligned}
\mathcal{L}_{\text {bos }}= & -\frac{1}{2} R-\frac{1}{4 \mathcal{C}^{2}} \partial^{m} \mathcal{C} \partial_{m} \mathcal{C}+\frac{1}{4 \mathcal{C}^{2}} \mathcal{B}^{m} \mathcal{B}_{m}+\frac{1}{8}\left(3 A^{m}-\frac{\mathcal{B}^{m}}{\mathcal{C}}\right)^{2} \\
& +\left[\frac{1}{2 \mathcal{C}}+\frac{b}{2}\left(2+\log \frac{8|h|^{2}}{\mathcal{C}}\right)\right]\left(D+\square \mathcal{C}+\frac{\mathcal{C}}{3} R\right) \\
& -\frac{|h|^{2}}{\mathcal{C}^{2}}-\frac{|\tilde{h}|^{2}}{2}-b\left(\tilde{h} h^{*}+\tilde{h}^{*} h+\frac{2|h|^{2}}{\mathcal{C}}\right)+i \frac{b}{2} \log \frac{h^{*}}{h} \partial_{m} \mathcal{B}^{m}
\end{aligned}
$$

where $R$ is the Ricci scalar and $A^{m}$ is the superconformal $U(1)$ gauge field; $A^{m}$ is an auxiliary field which becomes $A^{m}=\frac{\mathcal{B}^{m}}{3 \mathcal{C}}$ upon using its equations of motion. Finally, $\tilde{h}$ is a mixture of the auxiliary component $h_{\Sigma}$ of the chiral compensator and of $h$.

Actually comparison with the global case, eq.(2.11), shows that the only major modification comes from mixed contributions between this auxiliary field and $h$. The equation of motion for $D$, on the other hand, fixes the standard exponential behavior for $h$ in terms of the dilaton $\mathcal{C}$. Solving for $D$ and $\tilde{h}$ one obtains the following dilaton potential:

$$
V(\mathcal{C})=\frac{1}{8 e^{2}}\left(\frac{1}{\mathcal{C}}+2 b-2 b^{2} \mathcal{C}\right) e^{-\frac{1}{b \mathcal{C}}}
$$

very similar to the global case.

As before, the question of central interest is the origin of the kinetic terms for the effective degrees of freedom, which obviously do not appear in the simple terms that we have considered so far, eq. (3.5). We now turn to this problem.

\subsection{Kinetic terms for the composite degrees of freedom.}

The central object in our discussion will be the chiral superfield $\Pi$ introduced above in eq.(3.3). If we work in string units, then $V$ is of order $g^{0}$ and $(\Sigma \bar{\Sigma})^{3 / 2}$ is of order $g^{-2}$, where $g$ is the string coupling constant. Thus $\Pi$ is of order $g^{-2}$ as well and the term $\left.\Pi\right|_{F}$ which we used in the preceding subsection appears at the string tree level. 
Kinetic terms for the composite fields may be expected at the one-loop level. Indeed, one may construct from $\Pi$ the real superfield $\Pi \bar{\Pi} V /(\Sigma \bar{\Sigma})^{3}$ of conformal weight 2, whose D-component therefore is a natural candidate for a locally supersymmetric action term. Such a term is expected to appear at the string one loop-level (order $g^{0}$ when working in string units). As we will see momentarily, it naturally provides a kinetic term for the gauge and gaugino condensates, when interpreted in terms of the degrees of freedom present in the effective theory.

Moreover, higher weight generalized superpotential interactions [8] are expected on the same ground. They are again constructed from the superfield $\Pi$, the chiral projection of the vector superfield [7]. Indeed, the chiral superfield $\Sigma^{3}\left(\Pi / \Sigma^{3}\right)^{n}$ has conformal weight 3 and therefore leads, through its F-term, to a superpotential interaction. In string units, it is of order $g^{-2}$ and therefore all the corresponding interactions appear at the string tree level [8]. We will express them through a general function $\Sigma^{3} F\left[\Pi / \Sigma^{3}\right]$. The linear term in the expansion of $F(n=1)$ provides just the term $\left.\Pi\right|_{F}$ that was included in the action of the preceding subsection.

To recapitulate, we start with the more complete action:

$$
\mathcal{L}=\left.\alpha \frac{V}{(\Sigma \bar{\Sigma})^{3}} \Pi \bar{\Pi}\right|_{\mathrm{D}}-\left[\left.\left(\Sigma^{3} F\left(\frac{\Pi}{\Sigma^{3}}\right)+b \mathcal{P}[V] \ln \left(-2 \mathcal{P}[V] / \Sigma^{3}\right)\right)\right|_{\mathrm{F}}+\text { h.c. }\right]
$$

where $\alpha$ is some normalisation constant that we will take to be positive. We believe that this action represents the terms computable from string interactions that, interpreted in terms of the dynamical degrees of freedom, describe the effective theory below the condensation scale.

We could follow the same procedure as in the global case, that is, we could write the corresponding equations of motion for the different fields (in particular the 3 -form and the 2-form) and infer from them the expression for the potential energy in terms of the corresponding degrees of freedom. Being only interested here in this potential energy, we will depart somewhat from our original orientation and perform what amounts to a duality 
transformation in order to shorten the derivation.

Indeed, one can show that the same theory can be derived from the following Lagrangian:

$$
\begin{aligned}
\mathcal{L} & =\left.\left[\alpha \frac{V}{(\Sigma \bar{\Sigma})^{3}} \Pi \bar{\Pi}-\frac{(S+\bar{S})}{2} V-(X+\bar{X})\left(\frac{V}{2}\right)^{-1 / 2}(\Sigma \bar{\Sigma})^{3 / 2}\right]\right|_{\mathrm{D}} \\
& +\left(\left.\left[-\Sigma^{3} F\left(\frac{\Pi}{\Sigma^{3}}\right)+X \Pi+\frac{S U}{2}+\frac{b}{2} U \log \frac{U}{\Sigma^{3}}\right]\right|_{\mathrm{F}}+\text { h.c. }\right),
\end{aligned}
$$

where $S$ and $X$ are chiral superfields. Minimization with respect to $S$ ensures the constraint $U=-2 \mathcal{P}[V]$, whereas minimization with respect to $X$ ensures that $\Pi$ is given as in (3.3). Thus, $\Pi$ in (3.9) is to be taken as an independent chiral superfield.

Now, solving for $V$ and $U$ yields:

$$
\mathcal{L}=-\left.\frac{3}{2}(\Sigma \bar{\Sigma}) e^{-\frac{1}{3} K}\right|_{\mathrm{D}}+\left(\left.\Sigma^{3} W\right|_{\mathrm{F}}+\text { h.c. }\right)
$$

where the Kähler potential is given, up to a constant piece, by

$$
K=-\log (S+\bar{S}-\alpha \Pi \bar{\Pi})-2 \log (X+\bar{X})
$$

and the superpotential by

$$
W=X \Pi-F(\Pi)-\frac{b}{2 e} e^{-S / b}
$$

The field $\Pi / \Sigma^{3}$ has been redefined as $\Pi$.

The presence of new propagating superfields $X$ and $\Pi$ can be understood in the following way. The higher weight interactions [8] give rise to kinetic terms for fields that appear to be auxiliary at low energies, like the $h$ component of $V$. At high energies, these fields form full physical supermultiplets corresponding to superstring excitations. $X$ is an example of such a superfield, with its mass equal to the superstring mass scale. The superfield $\Pi$ contains the composite degrees of freedom of the gaugino bound state and its supersymmetric partners.

\footnotetext{
3 This can also be viewed from a description à la Nambu-Jona-Lasinio. As can be seen from (3.9), the field $X$ can be understood as an "auxiliary" field whose equation of motion ensures that $\Pi$ describes the gaugino condensate degrees of freedom. Similar couplings appear in a supersymmetric version of the Nambu-Jona-Lasinio scenario[9]. We wish to thank Yi-Yen Wu for pointing this out to us.
} 
With the theory now formulated in the standard supergravity framework, it is straightforward to obtain the scalar potential. As one can easily check, it suffers from an instability

as $S+\bar{S}-\alpha \Pi \bar{\Pi} \rightarrow 0^{+}$. However this is not a genuine problem of this approach and it can be cured in many ways. As an example, we include in the model a modulus $T$ which appears only in the Kähler potential. We therefore compute the scalar potential with

$$
K=-\log (S+\bar{S}-\alpha \Pi \bar{\Pi})-2 \log (X+\bar{X})-3 \log (T+\bar{T})
$$

and the superpotential of (3.12). It reads

$$
\begin{aligned}
V= & -\frac{1}{2 Y}|\Pi|^{2}+\frac{1}{Y(X+\bar{X})}(\Pi \bar{F}+\bar{\Pi} F) \\
+\frac{1}{Y(X+\bar{X})^{2}} & \left(3|F-X \Pi|^{2}+\frac{Y}{\alpha}\left|F^{\prime}-X\right|^{2}+\left[Y(S+\bar{S}+2 b)+3 b^{2}\right] e^{-\frac{S+\bar{S}}{b}-2}\right. \\
& \left.\quad-\left[e^{-\frac{S}{b}-1}\left((3 b+Y) \bar{F}-Y \bar{\Pi} \bar{F}^{\prime}+b \bar{\Pi}(X-2 \bar{X})\right)+\text { h.c. }\right]\right)
\end{aligned}
$$

where $Y=S+\bar{S}-\alpha \Pi \bar{\Pi}$ and $F^{\prime}=d F / d \Pi$.

In the simple case where the function $F$ is linear in $\Pi$, one can minimize with respect to all the fields but $s \equiv S+\bar{S}$ and $\rho \equiv|\Pi|$. The potential then has a smooth monotonic behaviour with respect to $s$ and $\rho$, going to $0^{+}$as any of these fields tends to infinity. There is no non-trivial minimum. On the other hand, there is no problem with generating the $\operatorname{Im} S$ axion mass: the Peccei-Quinn symmetry $S \rightarrow S+i a$ is broken by the superpotential terms involving $\Pi$ and the massive string superfield $X$. We reserve the study of more complete and realistic models to further work.

\section{Concluding remarks}

The vector supermultiplet formalism developed in this work allows a very natural effective action description of the coupled systems of gauge fields and linear supermultiplets, as present in the heterotic superstring compactifications. In particular, the vector component of this multiplet contains a three-form field that can describe either the field-strength 
of a massless Kalb-Ramond field or a massive axion, depending on the details of nonperturbative dynamics. Furthermore, the formalism allows straightforward incorporation of the higher-weight generalized superpotential interactions. In the equivalent dual description, the axion mass generation can be understood as an effect of non-perturbative superpotential terms that violate a Peccei-Quinn-like symmetry.

While (slowly) completing this article, we received a paper by C. Burgess, J.P. Derendinger, F. Quevedo and M. Quirós [10] where ideas similar to the ones presented here are developed, although with seemingly different motivations and only in the context of global supersymmetry. An earlier and somewhat different approach has been followed by Gaida and Lüst [1].

\section{Acknowledgments}

P.B. and T.R.T. would like to thank Theory Divisions at LBL and at CERN for their hospitality. P.B. also wishes to thank G. Girardi and R. Grimm for enlightening discussions on the 3 -form supermultiplet.

\section{References}

[1] G. Veneziano and S. Yankielowicz, Phys. Lett. B 113 (1982) 231; T.R. Taylor, Phys. Lett. B 164 (1985) 43; P. Binétruy and M.K. Gaillard, Phys. Lett. B 232 (1989) 82; S. Ferrara, N. Magnoli, T.R. Taylor and G. Veneziano, Phys. Lett. B 245 (1990) 409.

[2] S.J. Gates, Nucl. Phys. B 184 (1981) 381.

[3] G. Girardi and R. Grimm, Phys. Lett. B 260 (1991) 365.

[4] P. Binétruy, G. Girardi, R. Grimm and F. Pillon, paper in preparation.

[5] A. Aurilia and Y. Takahashi, Prog. of Theor. Phys. 66 (1981) 693. 
[6] A. Aurilia, Y. Takahashi and P.K. Townsend, Phys. Lett. B 95 (1980) 265.

[7] T. Kugo and S. Uehara, Nucl. Phys. B 222 (1983) 125; Nucl. Phys. B 226 (1983) 49.

[8] I. Antoniadis, E. Gava, K.S. Narain and T.R. Taylor, Nucl. Phys. B 432 (1994) 187.

[9] A. de la Macorra and G.G. Ross, Nucl. Phys. B 404 (1993) 321; E. Dudas, Ph. D. thesis, Orsay University (1994).

[10] C.P. Burgess, J.P. Derendinger, F. Quevedo and M. Quirós, Phys. Lett. B 348 (1995) 428.

[11] I. Gaida and D. Lüst, preprint HUB-IEP-94/33, hep-th/9412079. 